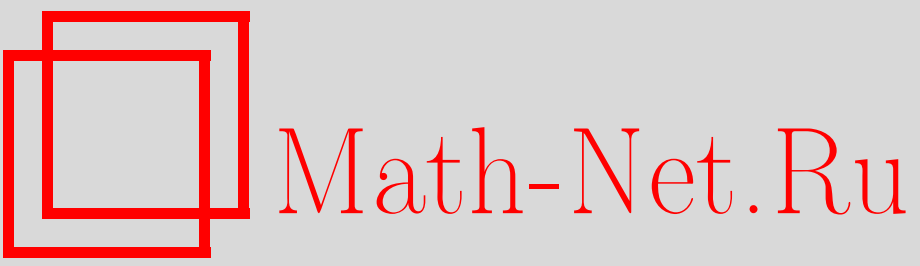

Г. А. Мартынов, Функции распределения бинарных растворов (точное аналитическое решение), ТМФ, 2000, том 123, номер 3, 500-515

DOI: https://doi.org/10.4213/tmf619

Использование Общероссийского математического портала Math-Net.Ru подразумевает, что вы прочитали и согласны с пользовательским соглашением http://www. mathnet.ru/rus/agreement

Параметры загрузки:

IP: 54.205 .225 .156

26 апреля 2023 г., $10: 27: 45$ 


\section{ФУНКЦИИ РАСПРЕДЕЛЕНИЯ БИНАРНЫХ РАСТВОРОВ (ТОЧНОЕ АНАЛИТИЧЕСКОЕ РЕШЕНИЕ)}

Показано, что обшее решение системы уравнений Орнштейна-Цернике для многокомпонентных растворов всегда может быть записано в виде $h_{\alpha \beta}=$ $\sum A_{\alpha \beta}^{j} \exp \left(-\lambda_{j} r\right) / r$, где $\lambda_{j}$ - корни трансцендентного уравнения $1-\rho \Delta\left(\lambda_{j}\right)=0, \mathrm{a}$ амплитуды $A_{\alpha \beta}^{j}$ определяются формулой, позволяюшей их рассчитать по известному значению прямых корреляционных функций. Исследованы свойства этого решения, в том числе поведение корней $\lambda_{j}$, и амплитуд $A_{\alpha \beta}^{j}$ трансцендентного уравнения в пределе малых плотностей и в окрестности критической точки. Установлен ряд соотношений для $A_{\alpha \beta}^{j}, C_{\alpha \beta}$. Получено уравнение состояния жидкости в окрестности критической точки, подтверждающее гипотезу подобия Ван-дер-Ваальса. Показано, что рассматриваемое разложение является асимптотическим в том отношении, что оно представляет собой разложение искомых функций в ряд по собственным функциям асимптотического уравнения Орнштейна-Цернике, справедливого при $r \rightarrow \infty$.

\section{ВВЕДЕНИЕ}

Как известно, в основе современной теории жидкостей лежит уравнение Орнштейна-Цернике (ОЦ), являюшееся строгим следствием основных постулатов статистической механики ${ }^{1)}$. Однако поскольку оно не замкнуто, его конкретное решение становится возможным только после присоединения к нему уравнения замыкания. К сожалению, точный вид последнего не известен. Поэтому до сих пор основные усилия ученых были направлены на поиск "хорошего" замыкания; анализу же самого уравнения ОЦ, как правило, не придавалось особого значения. Однако уравнение ОЦ является строгим следствием постулатов статистической механики, тогда как любое замыкание по своей природе является приближенным. Благодаря этому все полученные до сих пор решения системы “точное уравнение ОЦ плюс приближенное уравнение замыкания” также были приближенными.

\footnotetext{
1) См. [1], где уравнение ОЦ было получено путем тождественных преобразований распределения Гиббса, а также [2], где было показано, что уравнение ОЦ является точным решением иерархии ББГКИ.
}

${ }^{*}$ Институт физической химии РАН, Москва, Россия. E-mail:martynov@lmm.phyche.msk.su 
Наряду с поиском замыканий и построением приближенных решений представляет определенный интерес анализ тех свойств уравнения ОЦ, которые никак не связаны ни с замыканием, ни с конкретным видом потенциала взаимодействия. Полученные таким образом результаты можно считать точными, если только при их выводе не делалось каких-либо приближений. Рассмотрению точных следствий уравнения ОЦ и посвящена эта статья. В ней на основе системы уравнений ОЦ анализируются функции распределения бинарного раствора. Выбор бинарной смеси в качестве исходного объекта исследования обусловлен тем, что анализ уравнений для этой смеси не многим сложнее анализа уравнений для однокомпонентной жидкости; решение же уравнений для более сложных систем требует привлечения матричного анализа, что делает выкладки достаточно громоздкими. В то же время анализ бинарных смесей имеет то преимущество, что позволяет обнаружить ряд новых свойств уравнения ОЦ, не свойственных однокомпонентным жидкостям. В дальнейшем, конечно, полученные результаты должны быть обобщены на случай растворов, содержащих три и более компоненты. Ожидать при этом каких-то принципиально новых результатов или особых математических трудностей, по-видимому, не приходится. $\mathrm{K}$ тому же ряд заключений о поведении многокомпонентных растворов можно сделать уже сейчас путем экстраполяции данных, полученных для бинарных систем.

Мы начнем с преобразования уравнений ОЦ в ряд по собственным функциям асимптотического уравнения ОЦ (раздел 1), затем проанализируем трансцендентное уравнение, определяющее вид собственных функций (раздел 2), и рассмотрим амплитуды разложения (раздел 3 ). В разделе 4 будет дан вывод уравнения состояния для критической области, после чего мы перейдем к анализу газовых смесей и разбавленных растворов (раздел 5), а также к рассмотрению асимптотики функций распределения (раздел 6). В разделе 7 обсуждаются полученные результаты.

\section{1. ФУНКЦИИ РАСПРЕДЕЛЕНИЯ БИНАРНОЙ СМЕСИ}

В случае бинарной смеси, состоящей из компонент А и В, система уравнений ОЦ принимает вид [3]

$$
\begin{aligned}
h_{\mathrm{AA}}\left(r_{12}\right)-C_{\mathrm{AA}}\left(r_{12}\right)= & \rho\left\{\int_{V} C_{\mathrm{AA}}\left(r_{13}\right) h_{\mathrm{AA}}\left(r_{23}\right) d(3)+\int_{V} C_{\mathrm{AB}}\left(r_{13}\right) h_{\mathrm{BA}}\left(r_{23}\right) d(3)\right\}, \\
h_{\mathrm{AB}}\left(r_{12}\right)-C_{\mathrm{AB}}\left(r_{12}\right)= & \frac{1}{2} \rho\left\{\int_{V} C_{\mathrm{AA}}\left(r_{13}\right) h_{\mathrm{AB}}\left(r_{23}\right) d(3)+\int_{V} C_{\mathrm{BA}}\left(r_{13}\right) h_{\mathrm{AA}}\left(r_{23}\right) d(3)+\right. \\
& \left.+\int_{V} C_{\mathrm{AB}}\left(r_{13}\right) h_{\mathrm{BB}}\left(r_{23}\right) d(3)+\int_{V} C_{\mathrm{BB}}\left(r_{13}\right) h_{\mathrm{BA}}\left(r_{23}\right) d(3)\right\}, \\
h_{\mathrm{BB}}\left(r_{12}\right)-C_{\mathrm{BB}}\left(r_{12}\right)= & \rho\left\{\int_{V} C_{\mathrm{BA}}\left(r_{13}\right) h_{\mathrm{AB}}\left(r_{23}\right) d(3)+\int_{V} C_{\mathrm{BB}}\left(r_{13}\right) h_{\mathrm{BB}}\left(r_{23}\right) d(3)\right\},
\end{aligned}
$$

где $h_{\alpha \beta}=\sqrt{\nu_{\alpha}} \sqrt{\nu_{\beta}} \tilde{h}_{\alpha \beta}=\sqrt{\nu_{\alpha}} \sqrt{\nu_{\beta}}\left[\widetilde{G}_{\alpha \beta}-1\right], \widetilde{G}_{\alpha \beta}-$ двухчастичная функция распределения, $\tilde{h}_{\alpha \beta}$ - обшая корреляционная функция, $C_{\alpha \beta}=\sqrt{\nu_{\alpha}} \sqrt{\nu_{\beta}} \widetilde{C}_{\alpha \beta}, \widetilde{C}_{\alpha \beta}-$ прямая корреляционная функция, $\rho_{\alpha}-$ плотность компоненты $\alpha$ (каждый из греческих индек- 
сов $\alpha, \beta$ может принимать два латинских значения А и В) и

$$
\nu_{\alpha}=\frac{\rho_{\alpha}}{\rho}, \quad \nu_{\mathrm{A}}+\nu_{\mathrm{B}}=1, \quad \rho=\rho_{\mathrm{A}}+\rho_{\mathrm{B}}
$$

В дальнейшем перенормированные прямые корреляционные функции $C_{\alpha \beta}$, общую плотность вешества $\rho$ и мольные доли $\nu_{\alpha}$ будем считать заданными параметрами системы. Наша задача - найти общие корреляционные функции $h_{\alpha \beta}$, соответствующие этим параметрам.

В работе [3] было показано, что после перехода к фурье-образам

$$
\begin{aligned}
& h_{\alpha \beta}(k)=\int_{V} h_{\alpha \beta}(r) \exp (-i \mathbf{k r}) d \mathbf{r}=4 \pi \int_{0}^{\infty} h_{\alpha \beta}(r) \frac{\sin (k r)}{k r} r^{2} d r \\
& C_{\alpha \beta}(k)=\int_{V} C_{\alpha \beta}(r) \exp (-i \mathbf{k r}) d \mathbf{r}=4 \pi \int_{0}^{\infty} C_{\alpha \beta}(r) \frac{\sin (k r)}{k r} r^{2} d r
\end{aligned}
$$

система интегральных уравнений (1) сводится к системе трех алгебраических уравнений для общих корреляционных функций

$$
h_{\alpha \beta}(k)=\frac{\chi_{\alpha \beta}(k)}{1-\rho \Delta(k)},
$$

где

$$
\begin{gathered}
\chi_{\mathrm{AA}}(k)=\Delta(k)-C_{\mathrm{BB}}(k), \quad \chi_{\mathrm{AB}}(k)=C_{\mathrm{AB}}(k), \quad \chi_{\mathrm{BB}}=\Delta(k)-C_{\mathrm{AA}}(k), \\
\Delta(k)=C_{\mathrm{AA}}(k)+C_{\mathrm{BB}}(k)+\rho\left[C_{\mathrm{AB}}^{2}(k)-C_{\mathrm{AA}}(k) C_{\mathrm{BB}}(k)\right] .
\end{gathered}
$$

Используя обратное преобразование Фурье и равенства (4), получим²)

$$
\begin{aligned}
r h_{\alpha \beta}(r) & =\frac{1}{(2 \pi)^{3}} \int_{0}^{\infty} \frac{\chi_{\alpha \beta}(k)}{1-\rho \Delta(k)} \sin (k r) k d k= \\
& =\frac{1}{2(2 \pi)^{3}} \int_{-\infty}^{+\infty} \frac{\chi_{\alpha \beta}(k)}{1-\rho \Delta(k)} \sin (k r) k d k
\end{aligned}
$$

(здесь мы воспользовались тем, что $\chi_{\alpha \beta}$ и $\Delta$ зависят от модуля $k$ вектора $\mathbf{k}$ и что $\sin (k r) k$ является четной функцией $k$ ). Учитывая известное тригонометрическое соотношение $\sin (k r)=-i[\exp (i k r)-\cos (k r)]$ и то, что интеграл в (6) берется в симметричных пределах, $\operatorname{acos}(k r) k$ является нечетной функцией $k$, можно записать (6) в виде

$$
r h_{\alpha \beta}(r)=\frac{1}{2 i(2 \pi)^{3}} \int_{-\infty}^{+\infty} \frac{\chi_{\alpha \beta}(k) \exp (i k r)}{1-\rho \Delta(k)} k d k .
$$

Этот интеграл может быть взят с помощью теории вычетов. Для этого в интеграле (7) надо перейти от действительных значений $k$ к комплексным $k=\operatorname{Re} k+i \operatorname{Im} k$ и в комплексной плоскости замкнуть действительную ось дугой бесконечного радиуса. После

\footnotetext{
2) В последующих рассуждениях этого раздела мы следуем схеме, разработанной в [4] при анализе однокомпонентных систем.
} 
этого в верхней полуплоскости, ограниченной действительной осью $\operatorname{Re} k$ и проведенной дугой, надо найти значения полюсов подынтегрального выражения в (7). Пусть координаты этих полюсов задаются значениями $k_{j}=i z_{j}$, где $z_{j}=\lambda_{j}+i \mu_{j}$. В этом случае полюсы подьнтегрального выражения будут определяться корнями трансцендентного уравнения

$$
1-\rho \Delta\left(i z_{j}\right)=0 .
$$

В развернутом виде уравнение (8) записывается как (см. (4))

$$
1-\rho\left\{C_{\mathrm{AA}}\left(i z_{j}\right)+C_{\mathrm{BB}}\left(i z_{j}\right)+\rho\left[C_{\mathrm{AB}}^{2}\left(i z_{j}\right)-C_{\mathrm{AA}}\left(i z_{j}\right) C_{\mathrm{BB}}\left(i z_{j}\right)\right]\right\}=0,
$$

где

$$
\begin{aligned}
C_{\alpha \beta}\left(i z_{j}\right) & =4 \pi \int_{0}^{\infty} C_{\alpha \beta}(r) \frac{\sin \left(i z_{j} r\right)}{i z_{j} r} r^{2} d r=4 \pi \int_{0}^{\infty} C_{\alpha \beta}(r) \frac{\operatorname{sh}\left(z_{j} r\right)}{z_{j} r} r^{2} d r= \\
& =\frac{C_{\alpha \beta}^{*}\left(z_{j}\right)}{z_{j}} .
\end{aligned}
$$

Так как подынтегральное выражение в (7) имеет вид дроби $\xi / \psi$, где $\psi=1-\rho \Delta(k)$ и $\xi=k \chi_{\alpha \beta}(k) \exp (i k r)$, то по теореме о вычетах

$$
r h_{\alpha \beta}(r)=\frac{1}{2 \pi} \sum_{j} R_{j},
$$

где вычет в полюсе $j$ есть

$$
R_{j}=\frac{\xi\left(k_{j}\right)}{\left[\frac{d \psi(k)}{d k}\right]_{k_{j}}}=-\frac{k_{j} \chi_{\alpha \beta}\left(k_{j}\right) \exp \left(i k_{j} r\right)}{\rho \Delta^{\prime}\left(k_{j}\right)} .
$$

Здесь

$$
\begin{aligned}
\Delta^{\prime}\left(k_{j}\right)= & \frac{1}{i z_{j}}\left\{C_{\mathrm{AA}}^{\prime}\left(i z_{j}\right)\left[1-\rho C_{\mathrm{BB}}\left(i z_{j}\right)\right]+\right. \\
& \left.+C_{\mathrm{BB}}^{\prime}\left(i z_{j}\right)\left[1-\rho C_{\mathrm{AA}}\left(i z_{j}\right)\right]+2 \rho C_{\mathrm{AB}}\left(i z_{j}\right) C_{\mathrm{AB}}^{\prime}\left(i z_{j}\right)\right\}=\frac{\bar{\Delta}^{\prime}}{i z_{j}}
\end{aligned}
$$

$$
C_{\alpha \beta}^{\prime}\left(i z_{j}\right)=4 \pi \int_{0}^{\infty} C_{\alpha \beta}(r)\left[\operatorname{ch}\left(z_{j} r\right)-\frac{\operatorname{sh}\left(z_{j} r\right)}{z_{j} r}\right] r^{2} d r .
$$

Подставляя эти формулы в (11) и учитывая, что $\Delta\left(i z_{j}\right)=1 / \rho$, получим искомое выражение

$$
h_{\alpha \beta}(r)=\sum_{j} A_{\alpha \beta}^{j} \frac{\exp \left(-z_{j} r\right)}{r},
$$

в котором амплитуды

$$
A_{\alpha \beta}^{j}=-\frac{i z_{j} \chi_{\alpha \beta}\left(i z_{j}\right)}{2 \pi \rho^{2} \Delta^{\prime}\left(i z_{j}\right)}=\frac{z_{j}^{2} \chi_{\alpha \beta}\left(i z_{j}\right)}{2 \pi \rho^{2} \bar{\Delta}^{\prime}\left(i z_{j}\right)}
$$


где

$$
\chi_{\mathrm{AA}}\left(i z_{j}\right)=1-\rho C_{\mathrm{BB}}\left(i z_{j}\right), \quad \chi_{\mathrm{AB}}\left(i z_{j}\right)=\rho C_{\mathrm{AB}}\left(i z_{j}\right), \quad \chi_{\mathrm{BB}}\left(i z_{j}\right)=1-\rho C_{\mathrm{AA}}\left(i z_{j}\right) .
$$

Сделаем несколько замечаний по поводу полученных выражений.

Все проведенные выше преобразования являются строгими, т.к. по ходу выкладок никаких аппроксимаций не делалось. Поэтому ряд (15), в котором зависимость $h_{\alpha \beta}$ от $r$ выделена явно, полностью эквивалентен исходной системе интегральных уравнений (1). Как известно, последнюю можно считать определением неизвестных функций $h_{\alpha \beta}$ по заданным функциям $C_{\alpha \beta}$. Естественно, что и коэффициенты ряда (15) также можно рассчитать, только зная прямые корреляционные функции $C_{\alpha \beta}$ (см. (9) и (16)). В свою очередь, определить $C_{\alpha \beta}$ можно, только задав конкретный вид уравнения замыкания, связывающего между собой $h_{\alpha \beta}$ и $C_{\alpha \beta}$. Как мы увидим ниже, ряд (15) представляет собой разложение неизвестных функций $h_{\alpha \beta}$ в ряд по собственным функциям линейного уравнения ОЦ, описываюшего асимптотику функций распределения.

\section{2. АНАЛИЗ ТРАНСЦЕНДЕНТНОГО УРАВНЕНИЯ (9)}

В общем случае корни трансцендентного уравнения (9) могут быть как действительными, так и комплексными, поскольку по определению $z_{j}=\lambda_{j}+i \mu_{j}$. Поэтому, строго говоря, собственными функциями асимптотического уравнения ОЦ являются

$$
\frac{\exp \left(-\lambda_{j} r\right)}{r}, \quad \frac{\exp \left(-\lambda_{j} r\right) \sin \left(\mu_{j} r\right)}{r}, \quad \frac{\exp \left(-\lambda_{j} r\right) \cos \left(\mu_{j} r\right)}{r} .
$$

Соответственно общее решение (15) имеет вид

$$
\begin{aligned}
h_{\alpha \beta}(r) & =\sum_{j} \tilde{A}_{\alpha \beta}^{j} \frac{\exp \left(-\lambda_{j} r\right)}{r}+\sum_{j} \frac{\exp \left(-\lambda_{j} r\right)}{r}\left[\widetilde{B}_{\alpha \beta}^{j} \sin \left(\mu_{j} r\right)+\widetilde{C}_{\alpha \beta}^{j} \cos \left(\mu_{j} r\right)\right] \equiv \\
& \equiv \sum_{j} \tilde{A}_{\alpha \beta}^{j} \frac{\exp \left(-\lambda_{j} r\right)}{r}+\Psi(r) .
\end{aligned}
$$

В (18) постоянные $\tilde{A}_{\alpha \beta}^{j}$ определяются непосредственно формулами (16), т.к. благодаря тому, что $\lambda_{j}$ - действительные числа, $\tilde{A}_{\alpha \beta}^{j}=A_{\alpha \beta}^{j}$. При вычислении же постоянных $\widetilde{B}_{\alpha \beta}^{j}$ и $\widetilde{C}_{\alpha \beta}^{j}$ формулы (16) должны быть предварительно преобразованы так, чтобы в них исчезли мнимые компоненты [4]. Как известно, внутри твердого ядра частиц функции $h_{\alpha \beta}(r)=-1$. Этот эффект достигается в (18) за счет соответствуюшего подбора функции $\Psi(r)$ (т.е. за счет выбора коэффициентов $\widetilde{B}_{\alpha \beta}^{j}$ и $\widetilde{C}_{\alpha \beta}^{j}$ ). Таким образом, вторая сумма $\Psi(r)$ в (18) в основном описывает поведение корреляционных функций на малых расстояниях, тогда как первая сумма описывает асимптотические свойства корреляционных функций при $r \rightarrow \infty$. По сушеству, $\Psi(r)$ представляет собой слегка видоизмененньй тригонометрический ряд Фурье.

Если теперь подставить (18) в формулу для изотермической сжимаемости

$$
\kappa_{\theta}=\frac{1}{\rho \theta}\left\{1+4 \pi \rho \int_{0}^{\infty}\left[h_{\mathrm{AA}}(r)+h_{\mathrm{AB}}(r)+h_{\mathrm{BB}}(r)\right] r^{2} d r\right\}
$$


то получим

$$
\kappa_{\theta}=\frac{1}{\rho \theta}\left\{1+4 \pi \rho\left[\sum_{j} \frac{\tilde{A}_{j}}{\left(\lambda_{j}\right)^{2}}+\sum_{j}\left(\widetilde{B}_{j} \frac{2 \lambda_{j} \mu_{j}}{\left[\left(\lambda_{j}\right)^{2}+\left(\mu_{j}\right)^{2}\right]^{2}}+\widetilde{C}_{j} \frac{\left(\lambda_{j}\right)^{2}-\left(\mu_{j}\right)^{2}}{\left[\left(\lambda_{j}\right)^{2}+\left(\mu_{j}\right)^{2}\right]^{2}}\right)\right]\right\}
$$

где

$$
\tilde{A}_{j}=\tilde{A}_{\mathrm{AA}}^{j}+\tilde{A}_{\mathrm{AB}}^{j}+\tilde{A}_{\mathrm{BB}}^{j}, \quad \widetilde{B}_{j}=\widetilde{B}_{\mathrm{AA}}^{j}+\widetilde{B}_{\mathrm{AB}}^{j}+\widetilde{B}_{\mathrm{BB}}^{j}, \quad \widetilde{C}_{j}=\widetilde{C}_{\mathrm{AA}}^{j}+\widetilde{C}_{\mathrm{AB}}^{j}+\widetilde{C}_{\mathrm{BB}}^{j}
$$

Из (20) следует, что если $\lambda_{j} \rightarrow 0$, то вклад первой суммы в изотермическую сжимаемость неограниченно возрастает, тогда как вклад второй суммы всегда остается конечным, поскольку по определению $\mu_{j} \neq 0$. Поэтому поведение вешества в окрестности критической точки определяется в основном величиной постоянной $\lambda_{j}$.

Учитывая важное значение действительных корней, естественно задаться вопросом об их количестве. Для этого надо вернуться к трансцендентному уравнению (9). В него входят фурье-трансформанты прямых корреляционных функций, которые можно записать в виде (см. (10))

$$
C_{\alpha \beta}\left(i z_{j}\right)=\frac{C_{\alpha \beta}^{*}\left(z_{j}\right)}{z_{j}}
$$

где

$$
C_{\alpha \beta}^{*}\left(z_{j}\right)=4 \pi \int_{0}^{\infty} C_{\alpha \beta}(r) \operatorname{sh}\left(z_{j} r\right) r d r
$$

Подставляя эти выражения в $(9)$ и полагая $z_{j}=\lambda_{j}$, получим квадратное уравнение

$$
\lambda_{j}^{2}-\lambda_{j} \rho\left[C_{\mathrm{AA}}^{*}\left(\lambda_{j}\right)+C_{\mathrm{BB}}^{*}\left(\lambda_{j}\right)\right]-\rho^{2}\left[C_{\mathrm{AB}}^{*}\left(\lambda_{j}\right)-C_{\mathrm{AA}}^{*}\left(\lambda_{j}\right) C_{\mathrm{BB}}^{*}\left(\lambda_{j}\right)\right]=0,
$$

решение которого имеет вид

$$
2 \lambda_{1,2}=\rho\left\{C_{\mathrm{AA}}^{*}\left(\lambda_{j}\right)+C_{\mathrm{BB}}^{*}\left(\lambda_{j}\right) \pm \sqrt{\left[C_{\mathrm{AA}}^{*}\left(\lambda_{j}\right)-C_{\mathrm{BB}}^{*}\left(\lambda_{j}\right)\right]^{2}+4 C_{\mathrm{AB}}^{*}\left(\lambda_{j}\right)}\right\} .
$$

Поскольку правая часть (24) при заданных функциях $C_{\alpha \beta}(r)$ является однозначной функцией $\lambda_{j}$ (см. (22)), постольку трансцендентное уравнение (24) может иметь только два действительных решения $\lambda_{1,2}$, соответствуюших двум различным знакам перед корнем. При этом из уравнения со знаком минус следует, что

$$
C_{\mathrm{AB}}^{*}\left(\lambda_{2}\right) \rightarrow \sqrt{C_{\mathrm{AA}}^{*}\left(\lambda_{2}\right) C_{\mathrm{BB}}^{*}\left(\lambda_{2}\right)} \quad \text { при } \quad \lambda_{2} \rightarrow 0 .
$$

Заметим теперь, что при $\lambda_{2} \rightarrow 0$ в подынтегральном выражении в $\left(22^{\prime}\right) \operatorname{sh}\left(\lambda_{2} r\right) \rightarrow \lambda_{2} r$ и, следовательно,

$$
C_{\alpha \beta}^{*}\left(\lambda_{2}\right) \rightarrow 4 \pi \lambda_{2} \int_{0}^{\infty} C_{\alpha \beta}(r) r^{2} d r=\lambda_{2} C_{\alpha \beta}^{(2)},
$$

где

$$
C_{\alpha \beta}^{(2)}=4 \pi \int_{0}^{\infty} C_{\alpha \beta}(r) r^{2} d r
$$


- второй момент функции $C_{\alpha \beta}(r)$, который вообше не зависит от $\lambda_{2}$. Подставляя (26) в (25) и сокрашая на $\lambda_{2}$, получим

$$
C_{\mathrm{AB}}^{(2)}=\sqrt{C_{\mathrm{AA}}^{(2)} C_{\mathrm{BB}}^{(2)}} \quad \text { при } \quad \lambda_{2} \rightarrow 0 .
$$

В работах $[4,5]$ было показано, что если хотя бы один из действительных корней трансцендентного уравнения (8) стремится к нулю, то одновременно стремится к нулю и действительная часть всех остальных корней того же уравнения. Поэтому в критической точке корень $\lambda_{1}$, являюшийся решением уравнения (24) со знаком плюс, также равен нулю. Используя равенства (26) и (27), сведем уравнение (24) со знаком плюс к виду

$$
1-\rho\left(C_{\mathrm{AA}}^{(2)}+C_{\mathrm{BB}}^{(2)}\right)=0 .
$$

Эта формула является обобщением выражения $1-\rho C^{(2)}=0$, полученного ранее в работах $[5,6]$ для однокомпонентной системы. Очевидно, что в случае многокомпонентных систем

$$
1-\rho \sum_{\alpha} C_{\alpha \alpha}^{(2)}=0 .
$$

Интересно, что из этих равенств исчезают перекрестные функции $C_{\alpha \beta}, \alpha \neq \beta$. Это указывает на то, что в критической точке компоненты $\mathrm{A}, \mathrm{B}$ и т.д. ведут себя независимо друг от друга, т.е. так, как будто они не взаимодействуют между собой.

\section{3. АМПлИтУДЫ}

Рассмотрим теперь более детально формулы (16) для амплитуд. Учитывая конкретные значения функций $\chi_{\alpha \beta}$ (см. (4)) и то, что $\Delta\left(z_{j}\right)=1 / \rho$ при $z=z_{j}$, их можно переписать в виде

$$
A_{\mathrm{AA}}^{j}=A_{0}^{j}\left[1-\rho C_{\mathrm{BB}}\left(i z_{j}\right)\right], \quad A_{\mathrm{AB}}^{j}=A_{0}^{j} \rho C_{\mathrm{AB}}\left(i z_{j}\right), \quad A_{\mathrm{BB}}^{j}=A_{0}^{j}\left[1-\rho C_{\mathrm{AA}}\left(i z_{j}\right)\right],
$$

где

$$
A_{0}^{j}=\frac{z_{j}^{2}}{2 \pi \rho^{2} \bar{\Delta}^{\prime}\left(i z_{j}\right)} .
$$

Однако из (9) следует, что

$$
\rho C_{\mathrm{AB}}\left(i z_{j}\right)=\sqrt{\left[1-\rho C_{\mathrm{AA}}\left(i z_{j}\right)\right]\left[1-\rho C_{\mathrm{BB}}\left(i z_{j}\right)\right]} .
$$

Подставляя последнее равенство в формулу (30) для $A_{\mathrm{AB}}^{j}$, получим

$$
A_{\mathrm{AB}}^{j}=\sqrt{A_{\mathrm{AA}}^{j} A_{\mathrm{BB}}^{j}} .
$$

Это выражение похоже на формулу (27), а также на эмпирическое правило ЛоренцаБертло, предложивших в потенциале Леннард-Джонса для смесей

$$
\Phi_{\alpha \beta}=4 \varepsilon_{\alpha \beta}\left\{\left(\frac{\sigma}{r}\right)^{12}-\left(\frac{\sigma}{r}\right)^{6}\right\}
$$


считать, что

$$
\varepsilon_{\mathrm{AB}}=\sqrt{\varepsilon_{\mathrm{AA}} \varepsilon_{\mathrm{BB}}}, \quad \sigma_{\mathrm{AB}}=\frac{1}{2}\left(\sigma_{\mathrm{AA}}+\sigma_{\mathrm{BB}}\right) .
$$

По всей вероятности, именно этим совпадением и объясняется успех правила Лоренца-Бертло.

Из формул (30) также следует, что

$$
A_{\mathrm{AA}}^{j}=\frac{1-\rho C_{\mathrm{BB}}\left(i z_{j}\right)}{1-\rho C_{\mathrm{AA}}\left(i z_{j}\right)} A_{\mathrm{BB}}
$$

Поэтому в смесях величины $\rho C_{\mathrm{AA}}\left(i z_{j}\right)$ и $\rho C_{\mathrm{BB}}\left(i z_{j}\right)$ всегда меньше единицы.

\section{4. УРАВНЕНИЕ СОСТОЯНИЯ В КРИТИЧЕСКОЙ ОБЛАСТИ}

В этом разделе мы для простоты будем рассматривать однокомпонентные системы; обобщение на случай бинарных растворов элементарно, но приводит к довольно громоздким формулам.

Начнем с анализа формулы (19) для изотермической сжимаемости, которая в случае $\nu_{\mathrm{A}}=1, \nu_{\mathrm{B}}=0$ принимает вид

$$
\kappa_{\theta} \equiv \frac{1}{\rho}\left(\frac{\partial \rho}{\partial P}\right)_{\theta}=\frac{1}{\rho \theta}[1+\rho h(k=0)]=\frac{1}{\rho \theta\left[1-\rho C^{(2)}\right]},
$$

где $P$ - давление и $C^{(2)}(\rho, \theta)$ - второй момент прямой корреляционной функции $C(r ; \rho, \theta)$. Отсюда получаем

$$
\frac{\partial P}{\partial \rho}=\theta\left(1-\rho C^{(2)}\right), \quad \rho \frac{\partial^{2} P}{\partial \rho^{2}}=-\rho C^{(2)}-\rho \frac{\partial\left(\rho C^{(2)}\right)}{\partial \rho} .
$$

Покажем сначала, что из этих формул следует обычное вириальное разложение. С этой целью разложим функцию $C^{(2)}(\rho, \theta)$ в ряд по степеням $\Delta \rho=\rho-\rho_{0}$. В этом случае из первой формулы (38) следует, что

$$
\frac{\partial P}{\partial \rho}=\theta\left[1-\rho \sum_{m} \frac{\Delta \rho^{m}}{m !} \frac{\partial^{m} C^{(2)}\left(\rho_{0}, \theta\right)}{\partial \rho^{m}}\right] .
$$

Полагая затем $\rho_{0}=0$ и определяя вириальные коэффишиенты с помошью формулы

$$
B_{m+1}=-\frac{m+1}{(m+2) !} \frac{\partial^{m} C^{(2)}(0, \theta)}{\partial \rho^{m}}
$$

получим после интегрирования (39) по $\rho$ вириальный ряд

$$
P=\rho \theta\left[1+\sum_{m} \rho^{m} B_{m+1}(\theta)\right] .
$$


Подобное разложение можно проводить не только вокруг точки $\rho=0$, но и вообщевокруг любой точки фазовой диаграммы. Посмотрим, что будет, если в качестве таковой выбрать критическую точку.

Координаты критической точки $\rho_{\mathrm{c}}, \theta_{\mathrm{c}}$ определяются двумя равенствами

$$
\frac{\partial P}{\partial \rho}=0, \quad \frac{\partial^{2} P}{\partial \rho^{2}}=0
$$

С учетом (42) из (38) следует, что в критической точке

$$
\rho_{\mathrm{c}} C_{\mathrm{c}}^{(2)}=1, \quad \rho_{\mathrm{c}} \frac{\partial\left(\rho_{\mathrm{c}} C_{\mathrm{c}}^{(2)}\right)}{\partial \rho}=-1
$$

Введем безразмерные переменные

$$
q=\frac{\rho-\rho_{\mathrm{c}}}{\rho_{\mathrm{c}}}, \quad t=\frac{\theta-\theta_{\mathrm{c}}}{\theta_{\mathrm{c}}}, \quad p=\frac{P-P_{\mathrm{c}}}{\rho_{\mathrm{c}} \theta_{\mathrm{c}}} .
$$

Разлагая $C^{(2)}(\rho, \theta)$ по $q$ и $t$ и учитьвая равенства $(43)$, получим

$$
\begin{aligned}
\frac{\partial P}{\partial \rho}= & -\theta_{\mathrm{c}}\left\{\theta_{\mathrm{c}} \frac{\partial\left(\rho_{\mathrm{c}} C_{\mathrm{c}}^{(2)}\right)}{\partial \theta} t+\frac{1}{2} \rho_{\mathrm{c}}^{2} \frac{\partial^{2}\left(\rho_{\mathrm{c}} C_{\mathrm{c}}^{(2)}\right)}{\partial \rho^{2}} q^{2}+\right. \\
& \left.+\rho_{\mathrm{c}} \theta_{\mathrm{c}} \frac{\partial^{2}\left(\rho_{\mathrm{c}} C_{\mathrm{c}}^{(2)}\right)}{\partial \rho \partial \theta} q t+\frac{1}{2} \theta_{\mathrm{c}}^{2} \frac{\partial^{2}\left(\rho_{\mathrm{c}} C_{\mathrm{c}}^{(2)}\right)}{\partial \theta^{2}} t^{2}+\cdots\right\}
\end{aligned}
$$

Так как в критической точке функция $\rho C^{(2)}$ достигает своего максимального значения, равного 1 , то производные $\partial C^{(2)} / \partial \theta$ и $\partial^{2} C / \partial \rho^{2}$ всегда отрицательны. Поэтому

$$
\frac{\partial P}{\partial \rho}=\theta_{c}\left\{B_{t} t+3 B_{q q} q^{2}-2 B_{q t} q t-B_{t t} t^{2}-\cdots\right\},
$$

где $B_{t}, B_{q q}>0$, знак остальных коэффициентов не определен. Из (45) сразу получаем выражение для изотермической сжимаемости и уравнение состояния (последнее путем интегрирования равенства (45) по $q)$

$$
\begin{aligned}
\kappa_{\theta} & =\frac{\kappa_{\theta}^{\mathrm{id}}}{B_{t} t+3 B_{q q} q^{2}-2 B_{q t} q t-B_{t t} t^{2}-\cdots}, \\
p & =B_{t} q t+B_{q q} q^{3}-B_{q t} q^{2} t-B_{t t} q t^{2}-\cdots,
\end{aligned}
$$

где $\kappa_{\theta}^{\mathrm{id}}=1 /\left(\rho_{\mathrm{c}} \theta_{\mathrm{c}}\right)-$ сжимаемость идеального газа в критической точке.

Получим эти же выражения, но несколько другим путем. С этой целью обратимся к формуле (20) для изотермической сжимаемости и предположим, что справедливо неравенство

$$
4 \pi \rho \frac{A}{\lambda^{2}} \gg 1+4 \pi \rho \sum_{j}[\ldots] \quad \text { или } \quad \lambda_{\max } \equiv \sqrt{\frac{4 \pi \rho A}{1+4 \pi \rho \sum_{j}[\ldots]}} \gg \lambda .
$$




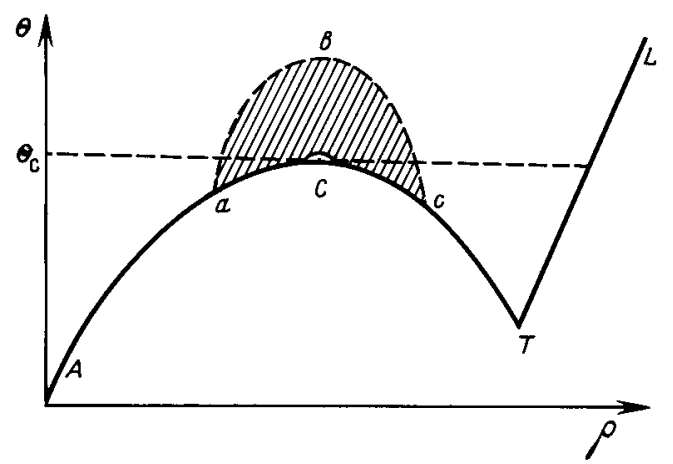

В этом случае изотермическая сжимаемость будет определяться выражением

$$
\kappa_{\theta}=\frac{4 \pi A}{\theta_{\mathrm{c}} \lambda^{2}}
$$

Так как условие (48) предполагает, что $\lambda$ достаточно мало, то в трансцендентном уравнении (9), которое в данном случае принимает вид

$$
1=4 \pi \rho \int_{0}^{\infty} C(r) \frac{\operatorname{sh}(\lambda r)}{\lambda r} r^{2} d r
$$

можно разложить $\operatorname{sh}(\lambda r)$ в ряд по $\lambda$ и ограничиться первым неисчезающим членом, пропорциональным $\lambda^{2}$. В результате получаем

$$
1-4 \pi \rho \int_{0}^{\infty} C(r) r^{2} d r=\lambda^{2} 4 \pi \rho \int_{0}^{\infty} C(r) r^{4} d r \quad \text { или } \quad \lambda^{2}=\frac{1-\rho C^{(2)}(\rho, \theta)}{\rho C^{(4)}(\rho, \theta)},
$$

где $C^{(4)}$ - момент четвертого порядка функции $C(r)$. В свою очередь, из формулы $(16)$ для амплитуды $A$, которая в случае однокомпонентных систем принимает вид

$$
A=\frac{\lambda^{2}}{2(2 \pi \rho)^{2} \int_{0}^{\infty} C(r)\left[\operatorname{ch}(\lambda r)-\frac{\operatorname{sh}(\lambda r)}{\lambda r}\right] r^{2} d r}
$$

следует, что при малых $\lambda$

$$
A=\frac{3}{2 \pi \rho C^{(4)}} .
$$

Подставляя (51) и (53) в (49), получим то же выражение (37) для $\kappa_{\theta}$, с которого мы начали. Таким образом, разложения (46) и (47) по $q$ и $t$ справедливы только при выполнении неравенства $\lambda_{\max } \gg \lambda$. С другой стороны, как было показано в статьях $[5,6]$, любое разложение в степенные ряды справедливо только вне области огромных флуктуаций с $\lambda_{\min }>\lambda \geqslant 0$, возникающей в непосредственной близости от критической точки. Таким образом, все полученные в этом разделе формулы справедливы в интервале

$$
\lambda_{\max } \gg \lambda \gg \lambda_{\min }
$$


Однако область существования полученных выражений определяется не только этими неравенствами. Если мы положим в $(46) q=0$, то обнаружим, что при $t<0$ изотермическая сжимаемость становится отрицательной. Но это противоречит распределению Гиббса, которое, как известно, справедливо только при $\kappa_{\theta} \geqslant 0$. В то же время возникшее противоречие находится в полном соответствии с результатами, полученными в $[5,6]$. По определению критическая точка лежит на вершине кривой фазового равновесия газ-жидкость; ниже ее находится двухфазная область, в которой газ и жидкость могут сосушествовать одновременно (см. рисунок). На рисунке изображена критическая область $a b c$ (заштрихована), в которой возможно разложение по $q$ и $t ; A C$ - кривая конденсации пара; $C T$ - кривая испарения жидкости; $T L$ - кривая кристаллизации жидкости. В двухфазной области зависимость распределения Гиббса от локальной плотности вешества $\rho$ исчезает. Соответственно и разложение функций распределения по $\rho$ становится невозможным. Поэтому, строго говоря, неравенства

$$
t \geqslant 0, \quad \theta \geqslant \theta_{\mathrm{c}}
$$

определяют нижнюю границу применимости полученных разложений. Но, с другой стороны, если область малых $\lambda$ простирается ниже критической изотермы $\theta_{\text {c }}$, то полученные выше разложения должны иметь аналитическое продолжение в эту область. Поэтому, скорее всего, их можно использовать и здесь (см. рисунок).

Если $t$ и $q$ достаточно малы, в (46) и (47) можно оставить первые два члена, что приводит к уравнениям

$$
\kappa_{\theta}=\frac{\kappa_{\theta}^{\mathrm{id}}}{B_{t} t+3 B_{q q} q^{2}}, \quad p=q\left(B_{t} t+B_{q q} q^{2}\right)
$$

Отсюда сразу следуют так называемые классические критические индексы $\gamma=-1$ и $\delta=3$. Действительно, из первого выражения получаем $\kappa_{\theta} \sim t^{-1}$ при $q=0$, а из второго $p \sim q^{3}$ при $t=0$.

Вводя новые переменные

$$
q^{\prime}=\sqrt{B_{q q}} q, \quad t^{\prime}=B_{t} t, \quad p^{\prime}=\sqrt{B_{q q}} p
$$

получим универсальные выражения для сжимаемости и для уравнения состояния

$$
\kappa_{\theta}=\frac{\kappa_{\theta}^{\mathrm{id}}}{t^{\prime}+3 q^{2}}, \quad p^{\prime}=q^{\prime}\left(t^{\prime}+q^{2}\right)
$$

Таким образом, согласно соотношениям (58) в окрестности критической точки приведенные термодинамические параметры всех жидкостей являются универсальными функииями плотности и температуры. Это находится в полном соответствии с гипотезой подобия Ван-дер-Ваальса. Однако из предыдушего следует, что эта гипотеза справедлива отнюдь не на всей фазовой диаграмме, как это предполагал ее автор, а только в критической области, где выполняются неравенства (54) (см. рисунок). 


\section{5. ГАЗОВЫЕ СМЕСИ И РАЗБАВЛЕННЫЕ РАСТВОРЫ}

В полученных формулах возможны два предельных перехода:

$$
\nu_{\mathrm{B}} \rightarrow 0, \quad \nu_{\mathrm{A}} \rightarrow 1 \text { при } \rho=\mathrm{const}
$$

(разбавленные растворы) и

$$
\rho \rightarrow 0 \text { при } \nu_{\alpha}=\text { const }
$$

(газовые смеси). Рассмотрим сначала первый случай.

Согласно выражениям (2) мольная доля $\nu_{\alpha}$ входит в уравнения в виде произведения соответствуюшей функции на $\sqrt{\nu_{\alpha}}$. Поэтому будем считать $\varepsilon=\sqrt{\nu_{\mathrm{B}}}$ малым параметром. В нулевом порядке по этому параметру получаем трансцендентное уравнение (50) и формулу для амплитуд (52) для однокомпонентной жидкости, состоящей только из молекул сорта A, поскольку в (9) и (16) при $\varepsilon=0$ все члены, имеющие индекс В, исчезают. $\mathrm{B}$ следуюшем приближении по $\varepsilon$ в решении, помимо $h_{\mathrm{AA}}$, появляется также функция

$$
h_{\mathrm{AB}}=\frac{z_{j}^{2} C_{\mathrm{AB}}\left(i z_{j}\right)}{2 \pi \rho C_{\mathrm{AA}}^{\prime}\left(i z_{j}\right)},
$$

величина которой пропорциональна $\varepsilon$. При этом значения констант $z_{j}$ по-прежнему определяются трансцендентным уравнением чистой жидкости (50). В приближении $\varepsilon^{2}=\nu_{\mathrm{B}}$ мы должны уже пользоваться полными уравнениями $(13),(15),(16)$ и т.д. Эти результаты вполне естественны и не требуют комментариев. Более интересным является случай газовой смеси.

Обычно при $\rho \rightarrow 0$ уравнение ОЦ (как, впрочем, и распределение Гиббса) решают путем разложения входящих в него функций в ряд по степеням $\rho$. Однако, если мы попытаемся разложить полученные выше формулы в ряд по $\rho$, то обнаружим, что это невозможно. Проще всего в этом убедиться, обратившись к трансцендентному уравнению (9), которое в данном случае удобно записать в виде

$$
\frac{\rho^{2} C_{\mathrm{AB}}^{2}\left(i z_{j}\right)}{\left[1-\rho C_{\mathrm{AA}}\left(i z_{j}\right)\right]\left[1-\rho C_{\mathrm{BB}}\left(i z_{j}\right)\right]}=1 .
$$

Если мы теперь положим в этом выражении все члены, пропорциональные $\rho$, равными нулю, то придем к бессмысленному выражению $0=1$. А если не сушествует нулевого приближения по $\rho$, то разложение в ряд по этому параметру невозможно. В то же время уравнению (62) можно удовлетворить при любом сколь угодно малом $\rho$, если предположить, что уменьшение плотности компенсируется соответствующим увеличением множителя $C_{\mathrm{AB}}\left(i z_{j}\right)$. Если обратиться к определению (10) функций $C_{\alpha \beta}\left(i z_{j}\right)$, легко заметить, что последние могут неограниченно возрастать при стремлении действительной части корня $\lambda_{j}$ к $\infty$, т.к. у них под знаком интеграла стоит отношение $\exp \left(\lambda_{j} r\right) / \lambda_{j}$. Это означает, что с уменьшением плотности газовой смеси радиус коррелящии

$$
R_{j}^{*}=\frac{1}{\lambda_{j}} \rightarrow 0 \quad \text { при } \quad \rho \rightarrow 0,
$$


причем это стремление происходит таким образом, что произведение $\rho C_{\alpha \beta}\left(i z_{j}\right)$ всегда остается конечным.

Посмотрим теперь, как ведут себя амплитуды $A_{\alpha \beta}^{j}$ при $\rho \rightarrow 0$. Вследствие того что произведения $\rho C_{\alpha \beta}\left(i z_{j}\right)$ всегда конечны, функции $\chi_{\alpha \beta}\left(i z_{j}\right)$ в (16) также ограничены. Соответственно ограничены сверху и произведения $\rho C_{\alpha \beta}^{\prime}\left(i z_{j}\right) \rightarrow \rho C_{\alpha \beta} / 2$. Поэтому поведение амплитуд при малых плотностях определяется отношением

$$
A_{\alpha \beta}^{j} \sim \frac{\lambda_{j}}{\rho} .
$$

Но ограниченность произведения $\rho C_{\alpha \beta}\left(i z_{j}\right)$ означает также, что сверху ограничено произведение

$$
\frac{\rho}{\lambda_{j}} C_{\alpha \beta}^{*}\left(i z_{j}\right)<\mathrm{const},
$$

где $C_{\alpha \beta}^{*}\left(i z_{j}\right)$ задано соотношением (10). Поэтому функция $C_{\alpha \beta}^{*}\left(i z_{j}\right)<\operatorname{const}\left(\lambda_{j} / \rho\right)$ неограниченно возрастает при $\lambda_{j} \rightarrow \infty$. В результате этого при $\rho \rightarrow 0$ все амплитуды неограниченно возрастают:

$$
A_{\alpha \beta}^{j} \rightarrow \infty \quad \text { при } \quad \rho \rightarrow 0 .
$$

Однако, если мы обратимся к формуле (20) для изотермической сжимаемости, то увидим, что в последнюю амплитуды входят в виде произведения

$$
\frac{\rho}{\lambda_{j}^{2}} A_{\alpha \beta}^{j} \sim \frac{\rho}{\lambda_{j}^{2}}\left(\operatorname{const} \frac{\lambda_{j}}{\rho}\right) \sim \frac{1}{\lambda_{j}},
$$

которое стремится к нулю при $\lambda_{j} \rightarrow \infty$. Поэтому

$$
\kappa_{\theta} \rightarrow \kappa_{\theta}^{\mathrm{id}}=\frac{1}{\rho \theta} \quad \text { при } \quad \rho \rightarrow 0
$$

Таким образом, точка $\rho=0$ является особой точкой для корреляционных функций из-за того, что в ней радиус корреляции обращается в нуль, а амплитуда - в бесконечность. В то же время с термодинамической точки зрения она является регулярной, т.к. в пределе $\rho \rightarrow 0$ параметры вещества плавно переходят в параметры идеального газа.

\section{6. АСИМПТОТИКА КОРРЕЛЯЦИОННЫХ ФУНКЦИЙ}

Утверждение о том, что точка нулевой плотности является особой точкой корреляционных функций, как будто противоречит хорошо известному факту, что в пределе малых плотностей [3]

$$
h\left(r_{12}\right)=f\left(r_{12}\right)+f\left(r_{12}\right) \int_{V} f\left(r_{13}\right) f\left(r_{32}\right) d \mathbf{r}_{3}+\cdots,
$$

где

$$
f(r)=\exp \left(-\frac{\Phi(r)}{\theta}\right)-1
$$


- функция Майера, $\theta=k_{\mathrm{B}} T$ - температура и $\Phi(r)$ - потенциал парного взаимодействия. Имеется ли здесь действительно какое-то противоречие, покажет более детальный анализ. Можно лишь указать, что в случае кулоновских систем, когда $\Phi \sim 1 / r$, формула (69) приводит к расходящемуся выражению для термодинамических функций [3]. В то же время переход к решению асимптотического уравнения Пуассона-Больцмана приводит к дебаевскому выражению для обшей корреляционной функции $h \sim \exp (-\lambda r) / r$, которое удовлетворяет всем требованиям задачи. Этот пример показывает: а) что в случае систем заряженных частиц возникают те же собственные функции, что и в случае нейтральных частиц (см. (15)), б) что эти функции порождаются асимптотикой соответствуюших уравнений, в) что ситуация в случае заряженных систем обратна той, которая возникает в случае нейтральных систем: вириальное разложение по $\rho$ расходится, а асимптотическое разложение (15), наоборот, устраняет расходимость. Таким образом, асимптотические разложения имеют право на сушествование. Все это заставляет еше раз обратиться к анализу асимптотики функций распределения системы нейтральных частиц.

Будем искать решение системы уравнений ОЦ (1) в виде $h_{\alpha \beta}=A_{\alpha \beta} \exp (-\lambda r) / r$, где $\lambda$ пока что произвольная постоянная. Предположим, что выполняется условие

$$
\frac{C_{\alpha \beta}(r)}{h_{\alpha \beta}(r)} \sim C_{\alpha \beta}(r) r e^{\lambda r} \rightarrow 0 \quad \text { при } \quad r \rightarrow \infty .
$$

В этом случае в левой части уравнений (1) на больших расстояниях можно пренебречь прямыми корреляционными функциями $C_{\alpha \beta}(r)$ по сравнению с обшими корреляционными функциями $h_{\alpha \beta}(r)$.

Рассмотрим теперь интегралы, стояшие в правой части системы (1),

$$
\begin{aligned}
\int_{V} C_{\alpha \beta}\left(r_{13}\right) h_{\beta \gamma}\left(r_{23}\right) d \mathbf{r}= & 2 \pi\left\{\int_{0}^{r} C_{\alpha \beta}(x) x d x \int_{r-x}^{r+x} h_{\beta \gamma}(y) y d y+\right. \\
& \left.+\int_{r}^{\infty} C_{\alpha \beta}(x) x d x \int_{x-r}^{x+r} h_{\beta \gamma}(y) d y\right\} .
\end{aligned}
$$

Подставим в них $h_{\alpha \beta}=A_{\alpha \beta} \exp (-\lambda r) / r$, после чего они примут вид

$$
4 \pi A_{\beta \gamma}\left\{e^{-\lambda r} \int_{0}^{r} C_{\alpha \beta}(x) \frac{\operatorname{sh}(\lambda x)}{\lambda x} x^{2} d x+\frac{\operatorname{sh}(\lambda r)}{\lambda} \int_{r}^{\infty} C_{\alpha \beta}(x) e^{-\lambda x} x d x\right\} .
$$

С помошью условия (70) легко показать, что в пределе больших расстояний второй интеграл в (72) обрашается в нуль, и, следовательно, при $r \rightarrow \infty$ имеем

$$
\begin{aligned}
\int_{V} C_{\alpha \beta}\left(r_{13}\right) h_{\beta \gamma}\left(r_{23}\right) d \mathbf{r} \rightarrow & \\
& \rightarrow A_{\beta \gamma} e^{-\lambda r} 4 \pi \int_{0}^{\infty} C_{\alpha \beta}(x) \frac{\operatorname{sh}(\lambda x)}{\lambda x} x^{2} d x=A_{\beta \gamma} e^{-\lambda r} C_{\alpha \beta}(\lambda) .
\end{aligned}
$$


Подставляя эти выражения в уравнения (1), опуская в левой части $C_{\alpha \beta}$ и сокрашая на $\exp (-\lambda r)$, получим алгебраическую систему трех однородных уравнений для определения трех неизвестных постоянных $A_{\alpha \beta}$ :

$$
\begin{aligned}
\left(1-\rho C_{\mathrm{AA}}\right) A_{\mathrm{AA}}+\rho C_{\mathrm{AB}} A_{\mathrm{AB}} & =0, \\
\rho C_{\mathrm{AB}} A_{\mathrm{AA}}+\left(2-\rho C_{\mathrm{AA}}-\rho C_{\mathrm{BB}}\right) A_{\mathrm{AB}}+\rho C_{\mathrm{AB}} A_{\mathrm{BB}} & =0, \\
\rho C_{\mathrm{AB}} A_{\mathrm{AB}}+\left(1-\rho C_{\mathrm{BB}}\right) A_{\mathrm{BB}} & =0 .
\end{aligned}
$$

Эта система может иметь решение только при условии, что детерминант, составленный из коэффициентов этих уравнений, равен нулю. Произведя соответствуюшие вычисления, приходим к трансцендентному уравнению (9), которое, таким образом, имеет смысл равенства нулю указанного детерминанта. Другими словами, трансцендентное уравнение (9) имеет смысл условия сушествования решения при больших $r$. Так как трансцендентное уравнение имеет бесконечное число корней, то соответственно и асимптотическая система уравнений ОЦ (74) имеет бесконечное число собственных функций (15). Но, конечно, определить амплитуды $A_{\alpha \beta}$ из асимптотической системы уравнений невозможно; последнее можно сделать только с помошью формул (16), если известен конкретньй вид прямых корреляционных функций $C_{\alpha \beta}(r)$, заданных на малых расстояниях.

Из полученных формул также следует, что асимптотика всех функций распределения $h_{\alpha \beta}$ одинакова независимо от сорта частиц. Этот вывод подтверждается непосредственными расчетами [4].

\section{7. ОБСУЖДЕНИЕ}

До сих пор существовал только один способ аналитического решения уравнений ОЦ (и эквивалентного ему распределения Гиббса) - разложение в вириальные ряды по степеням плотности вешества $\rho$. Как известно, первый член этого ряда описывает состояние идеального газа, при котором частицы вообше не взаимодействуют между собой, второй учитывает парные взаимодействия, третий - тройные и т.д. Очевидно, что чем больше мы учтем членов вириального ряда, тем более “коллективным" будет найденное решение, тем на бо́льших расстояниях от данной частицы мы сможем обнаружить то возмушение, которое эта частица вносит в окружающую среду. Таким образом, вириальное разложение предполагает, что мы постепенно движемся от малых расстояний к большим. Однако из-за технических трудностей удается вычислить всего лишь несколько первых членов вириального ряда, и, следовательно, до действительно больших расстояний (т.е. до асимптотики) продвинуться практически невозможно. Но в то же время найденных членов, как правило, бывает достаточно для вычисления термодинамических функций разреженных газов; для вычисления термодинамических функций жидкостей метод вириальных разложений вообше неприменим, т.к. в “жидкой” области ряды расходятся [6].

Выше было показано, что наряду с вириальным возможно также асимптотическое разложение функций распределения. В основе его лежит хорошо известный факт, что чем дальше мы отходим от данной частицы, тем меньше создаваемое ею возмушение 
окружающей среды. Вследствие этого вне корреляционной сферы все функции распределения равны единице. Отклонение от единицы можно считать своеобразным малым параметром задачи. Чтобы воспользоваться им, надо в уравнении ОЦ перейти к большим расстояниям и учесть малость прямой корреляционной функции (см. (70)). Пренебрегая последней, получим систему линейных уравнений ОЦ, по собственным функциям которой можно разложить общее решение. $\mathrm{K}$ тому же разложению можно прийти и формальным способом с помошью фурье-преобразования уравнения ОЦ (см. раздел 1). Асимптотический способ решения является столь же обшим, как и разложение в вириальные ряды, поскольку и в том, и в другом случаях исходное состояние вещества не зависит от вида потенииала взаимодействия (при вириальном разложении это достигается тем, что в состоянии идеального газа всякое взаимодействие между частицами исчезает; при асимптотическом разложении - тем, что вне корреляционной сферы всегда царит абсолютный хаос, при котором все функции распределения $G_{\alpha \beta}=1$ ). Однако асимптотический подход несколько шире вириального, т.к. обращение функции распределения в единицу справедливо всегда как в жидкостях, так и в газах, тогда как вириальное разложение справедливо только в случае разреженных газов.

Данная статья посвящена исследованию асимптотического решения уравнения ОЦ. В ней мы установили некоторые математические особенности асимптотического разложения (15) и получили ряд формул, определяющих поведение декрементов затухания $\lambda_{j}$ и амплитуд разложения $A_{\alpha \beta}^{j}$ (см. формулы $\left.(27),(33)\right)$. Так как асимптотический метод учитывает взаимодействие сразу всех частиц системы, его применение наиболее плодотворно при описании тех состояний вешества, в которых большие расстояния играют решающую роль. В первую очередь это относится к критическим явлениям $[5,6]$. В частности, выше на основе асимптотического разложения были получены выражение для изотермической сжимаемости и уравнение состояния вещества в критической области (58). Универсальность этих выражений подтверждает гипотезу подобия Ван-дер-Ваальса, согласно которой в окрестности критической точки безразмерное уравнение состояния для всех жидкостей имеет один и тот же вид.

\section{Список литературы}

[1] T. Morita, K. Hiroike. Progr. Theor. Phys. 1960. V. 23. P. 1003; V. 24. P. 317; 1961. V. 25. P. 537.

[2] Г. А. Мартынов. ТМФ. 1975. Т. 22. № 1. C. 85; G. A. Martynov. Mol. Phys. 1981. V. 42. P. 329.

[3] G. A. Martynov. Fundamental Theory of Liquids. Method of Distribution Function. Bristol, Philadelphia, New York: Adam Hilger, 1992.

[4] R. Evans, R. J. Leote de Carvalho, D. C. Hoyyle. J. Chem. Phys. 1994. V. 100. P. 591.

[5] Г. А. Мартынов. ТМФ. 1999. Т. 119. № 3. С. 498.

[6] Г. А. Мартынов. УФН. 1999. Т. 169. № 6. С. 595.

Поступила в редакцию 11.XI.1999 г. 